\title{
Iterating Registration and Activation Detection to Overcome Activation Bias in fMRI Motion Estimates
}

\author{
Jeff Orchard* and M. Stella Atkins* \\ Simon Fraser University, Burnaby BC V5A 1S6, Canada, \\ $\{j$ jo, stella\}@cs.sfu.ca
}

\begin{abstract}
Most intensity-based fMRI registration methods do not account for the fact that the volumes being aligned may differ: one may have blood oxygen level dependent (BOLD) contrast while the other does not. Especially in least-squares registration, this can result in motion parameter errors that are correlated to the stimulus. An iterative technique to overcome this activation bias is proposed and analyzed. The method, using mostly off-the-shelf software, is able to find the least-squares solution to both the registration and activation detection problems simultaneously. The resulting motion parameters and activation maps are considerably more accurate, yielding two-thirds fewer false-positive and one-third fewer false-negative activations.
\end{abstract}

\section{Introduction}

In functional MRI (fMRI), patient motion can have a very damaging effect on the accuracy of the resulting statistical parametric activation maps. Patient movements of a fraction of a millimetre or degree have the potential to cause falsenegative activations [1] and false-positive activations [2].

A host of registration techniques have been proposed to combat patient motion [1,34. These methods use a variety of cost functions and optimization schemes. One commonly-used cost function is the least-squares cost function, which can be shown to be the optimal choice when the images being compared differ only by Gaussian noise. However, least-squares techniques are sensitive to outliers. In fMRI, outliers come from a variety of sources, including the blood oxygen level dependent (BOLD) signal itself. Freire et al. [5] showed that the presence of BOLD contrast can bias the motion estimates calculated by least-squares registration methods. This influence becomes noticeable in fMRI datasets acquired on scanners with field strengths of 3 Tesla and higher. When the dataset is motion-compensated with these inaccurate motion estimates, stimulus-correlated motion is introduced into the dataset. Stimulus-correlated motion is especially damaging [2] because it makes it impossible for the activation detection algorithm to distinguish between intensity changes caused by

\footnotetext{
* This work was supported in part by the Natural Science and Engineering Research Council of Canada (NSERC)
} 
motion and those caused by activation. The activation detection process then interprets signal changes due to motion as activation, and yields a high number of false-positive activations. Furthermore, the added motion can decrease the significance of the true activation, resulting in false-negatives.

A natural approach to overcome activation bias is to iterate registration and activation detection with the hope that the final result will reflect both a minimum in the registration problem and a minimum in the activation problem. For example, one could perform motion correction first, and then run activation detection on the "aligned" dataset. After removing the estimated activation, the process could be repeated until subsequent iterations yield no change and the iterative method converges. This is a form of fixed-point iteration, which we will refer to as paired iteration. Unfortunately, this method rarely yields the expected motion parameters and activation maps. Instead, the activation maps often contain brain-edge artifacts, and the motion parameters often contain large stimulus-correlated components.

A variation of this approach has been proposed [6]. Following an initial registration step, activation detection is performed. The resulting activation map is thresholded and dilated to form a binary activation mask. Voxels under the mask are considered to be potentially active and are excluded from a second registration step, thereby shifting the weight of the registration process onto the unmasked portion of the volume. While this method has shown good results on both simulated and in vivo datasets, fMRI experiments that contain large regions of activation may cause the method to be less accurate, since the registration must then rely on information from fewer voxels. Also, the binary inclusion/exclusion of voxels may cause the method to be unstable.

The Simultaneous Registration and Activation (SRA) model [7] was designed to address the interdependence between motion and activation. It combines the two problems into a single least-squares problem, allowing the solution of both motion and activation simultaneously. The solution offered in 7 uses matrix $\mathrm{QR}$ decomposition and is implemented in $\mathrm{C}++$. For the sake of consistency, however, it is desirable to use one's current software to solve the SRA model rather than to use this new piece of software.

In this paper, we show that by introducing a simple adjustment step, the paired iteration approach described above can solve the SRA model and yield exactly the same solution as the QR decomposition approach. We explain the links between the QR solution and the paired iteration solution, and describe an algorithm that uses almost entirely off-the-shelf software to accurately resolve the motion/activation interdependency.

\section{Combined Registration and Activation Model}

To solve for both motion and activation simultaneously, a single model has to include the effect of both. In [7, this combined model is refered to as the Simultaneous Registration and Activation (SRA) model, and takes the form

$\{$ dataset $\} \approx\{$ baseline $\}+\{$ motion $\}+\{$ activation $\}$ 


$$
\mathbf{F} \approx \mathbf{G}+\mathbf{A X}+\mathbf{Y B}
$$

in which the matrix $\mathbf{F}$ stores one volume from the dataset in each column, $\mathbf{A X}$ is the linearization of the 3D (six-degree-of-freedom) rigid-body motion transformation, A holds the partial derivatives of the reference volume with respect to the motion parameters (in columns), and $\mathbf{X}$ holds the time series for the six motion parameters (one parameter per row). The term YB is the activation term in which $\mathbf{Y}$ holds $s$ activation maps (one in each column), and each row of $\mathbf{B}$ holds one of the $s$ time-series regressors for the activation detection general linear model (GLM).

A method to solve the SRA model was proposed in [7]. The solution is an iterative method that uses matrix QR decomposition to find a least-squares solution for the motion and the activation map simultaneously.

However, the least-squares solution to (1) is not unique. It can be shown that if $(\mathbf{X}, \mathbf{Y})$ is a solution, then the solution space is

$$
\left\{(\mathbf{X}+\alpha \mathbf{B}, \mathbf{Y}-\mathbf{A} \alpha) \mid \forall \alpha \in \mathbb{R}^{6 \times s}\right\},
$$

where $\alpha$ is a small, arbitrary matrix used to span the $6 s$-dimensional space. That is, $(\mathbf{X}+\alpha \mathbf{B}, \mathbf{Y}-\mathbf{A} \alpha)$ yields an equivalent solution for any $\alpha$.

This non-uniqueness is the reason that the paired iteration approach described above does not yield a satisfactory answer. While the paired iteration method does, indeed, yield a least-squares solution, the solution can be any one of the solutions in (2), most of which are not relevant to fMRI analysis. To get around this issue, the QR decomposition method uses an additional constraint in which a specifically-chosen cost function is minimized during each iteration. The constraint favours an activation map that has very low intensities everywhere except for where activation occurs. This characteristic is called "sparsity". We can find the solution with the greatest sparsity by finding the $\alpha$ that solves

$$
\min _{\alpha}\left(\sum_{i}\left|\mathbf{Y}_{i}-[\mathbf{A} \alpha]_{i}\right|\right),
$$

where $\mathbf{Y}_{i}$ and $[\mathbf{A} \alpha]_{i}$ refer to the $i^{\text {th }}$ voxels in $\mathbf{Y}$ and $\mathbf{A} \alpha$, respectively. Simply put, we wish to find the $\alpha$ that minimizes the $\ell_{1}$ norm of the adjusted activation map. In doing so, we remove edge artifacts from the initial activation map. In the following section, we adopt the same strategy for the paired iteration approach.

\section{Constrained Paired Iteration}

The paired iteration algorithm can be implemented both with and without the additional constraint in (3). We will refer to these algorithms as constrained paired iteration (CPI) and unconstrained paired iteration (UPI), respectively.

For the CPI method, we apply the constraint after each iteration. In this way, not only does the process converge to a least-squares solution of (11), but the solution also satisfies the additional constraint in (3). Figure 1 depicts the 


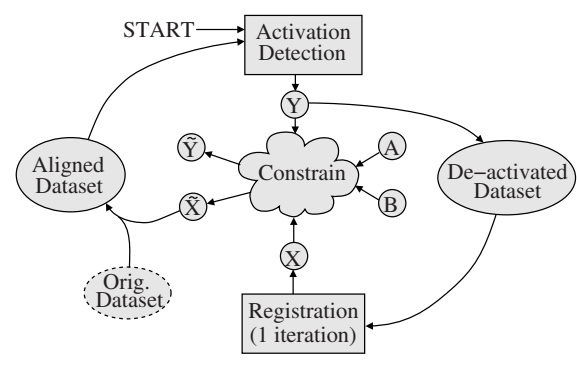

(a)

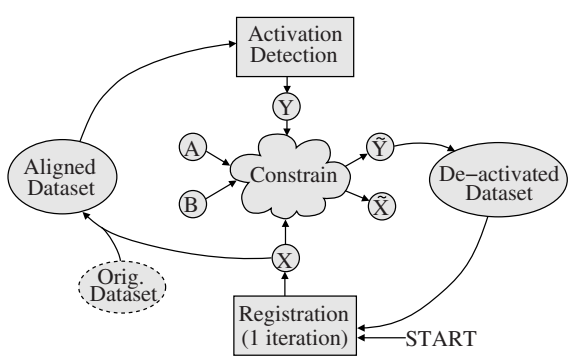

(b)

Fig. 1. Constrained paired iteration flowcharts. In (a), activation detection is performed first, while in (b), registration is performed first. Both methods converge to the same solution.

CPI method graphically. In Fig. 1(a), the activation detection is performed first yielding an estimate for the activation map $\mathbf{Y}$, followed by a single iteration of the registration method, yielding an estimate for the motion $\mathbf{X}$. The resulting motion and activation estimates are then fed into the constraint. After the constraint is applied, the adjusted motion estimates, $\tilde{\mathbf{X}}$, are combined with the current best-guess set of motion parameters (initially set to zero), and the original dataset is resampled. Another approach to the paired iteration method is to start the process with registration, followed by activation detection, as depicted in Fig. 1(b)

The CPI method in Fig. 1(a) is mathematically equivalent to the QR decomposition method. For each iteration, both methods produce an initial, unconstrained $(\mathbf{X}, \mathbf{Y})$ solution such that the rows of $\mathbf{X}$ (the time series for the six motion parameters) are orthogonal to the rows of $\mathbf{B}$ (the time-series regressors). Since the unconstrained $\mathbf{X}$-solutions match, the $\mathbf{Y}$-solutions must also match. Naturally, after applying the constraint, the two methods yield the same constrained solution as well.

To see that the unconstrained motion estimates of the CPI method in Fig. 1(a) are orthogonal to the rows of $\mathbf{B}$, consider the following. After performing activation detection without any motion correction, and removing this estimated activation from the dataset, the resulting voxel time series must all be orthogonal to the activation regressors stored in the rows of $\mathbf{B}$. This limits the possible solutions for the registration method to only those that are also orthogonal to the stimulus regressors. Hence, the time series of each motion parameter must be orthogonal to all the regressors in the activation detection GLM. 


\section{Methods}

Both the constrained and unconstrained paired iteration methods were implemented and tested. The implementation consists of a Perl (Practical Extraction and Report Language) script that makes the appropriate calls to command-line programs of the AFNI (Analysis of Functional NeuroImages) package [8]. AFNI is a suite of highly efficient programs for processing fMRI datasets, and includes almost all the necessary functions required for the paired iteration methods proposed here.

Only two steps were implemented using our own $\mathrm{C}++$ code. We used a $\mathrm{C}++$ program to combine the motion increment with the current best-guess. However, the motion increment can easily be added to the best-guess using simple Perl functions. Also, we used a $\mathrm{C}++$ program to implement the additional constraint. The constraint program reads in the initial ( $\mathbf{X}, \mathbf{Y}$ ) solution (as well as $\mathbf{A}$ and $\mathbf{B}$ ) and outputs the constrained solution $(\tilde{\mathbf{X}}, \tilde{\mathbf{Y}})$. The code uses the Nelder-Mead downhill simplex method [9] from Numerical Recipes in $C$ [10] to perform the minimization search.

For both the CPI and UPI methods, we have the choice of starting the iteration with registration or activation detection. For notational purposes, we will distinguish between the two by adding a suffix to the acronym: an " $\mathrm{R}$ " for registration first, or an "A" for activation detection first. Thus, the CPI method that begins with activation detection is denoted "CPI-A". In addition to these four methods, we also tested the QR decomposition method (denoted "QR"), as well as the standard sequential method (denoted "Std") in which a full leastsquares registration method is run once, followed by activation detection.

To test the performance of these six algorithms, simulated fMRI datasets containing 40 frames with known activation and motion were created. An original EPI dataset with dimensions $64 \times 64 \times 30$ (field of view of $240 \times 240 \times 120 \mathrm{~mm}$ ) was filtered with a $3 \times 3 \times 3$ median filter, and then duplicated 40 times. A $5 \%$ BOLD contrast was then (optionally) added to frames 5 through 15, and 25 through 35. The region of activation was specified by an activation template that was manually drawn over portions of the occipital and parietal regions of the brain, covering a total of about $13 \%$ of the brain. Motion was then added to the dataset. Resampling was done using Fourier interpolation [11]12]. Finally, Gaussian noise with a standard deviation of $2.5 \%$ was added to the dataset, followed by spatial smoothing using a Gaussian kernel with a full-width at half-maximum of $5 \mathrm{~mm}$.

The 40 simulated datasets were generated with varying mixtures of activation and motion. Ten datasets were generated for each of the following four scenarios:

1. The dataset has activation and random motion.

2. The dataset has activation and stimulus-correlated random motion.

3. The dataset has stimulus-correlation random motion, but no activation.

4. The dataset has activation, but no motion.

For each simulated dataset, the six analysis methods were used to produce activation maps. These activation maps contain both a linear fit coefficient and a correlation coefficient. A binary activation mask was then created by including 
voxels for which the linear fit coefficient was greater (in magnitude) than 20, and the correlation coefficient was greater (in magnitude) than 0.505 (corresponding to significance $p=0.001$ ). A true activation mask was also created, but from a dataset that was perfectly aligned. Comparing each activation mask to the true activation mask allowed the tabulation of false-positive and false-negative activation counts.

\section{Results}

In Fig. 2, the errors in the motion parameters calculated by the paired iteration methods are plotted with those of the standard least-squares method. The results from the two CPI methods (which converged in less than 2 minutes on a $1.2 \mathrm{GHz}$ AMD Athlon computer) are almost indistinguishable from each other. Although the QR decomposition solution is not shown, its motion estimates are very close to those of the two CPI methods. Note that the stimulus-correlated motion errors that appear in the standard and UPI estimates are not present in the motion estimates of the two CPI methods.
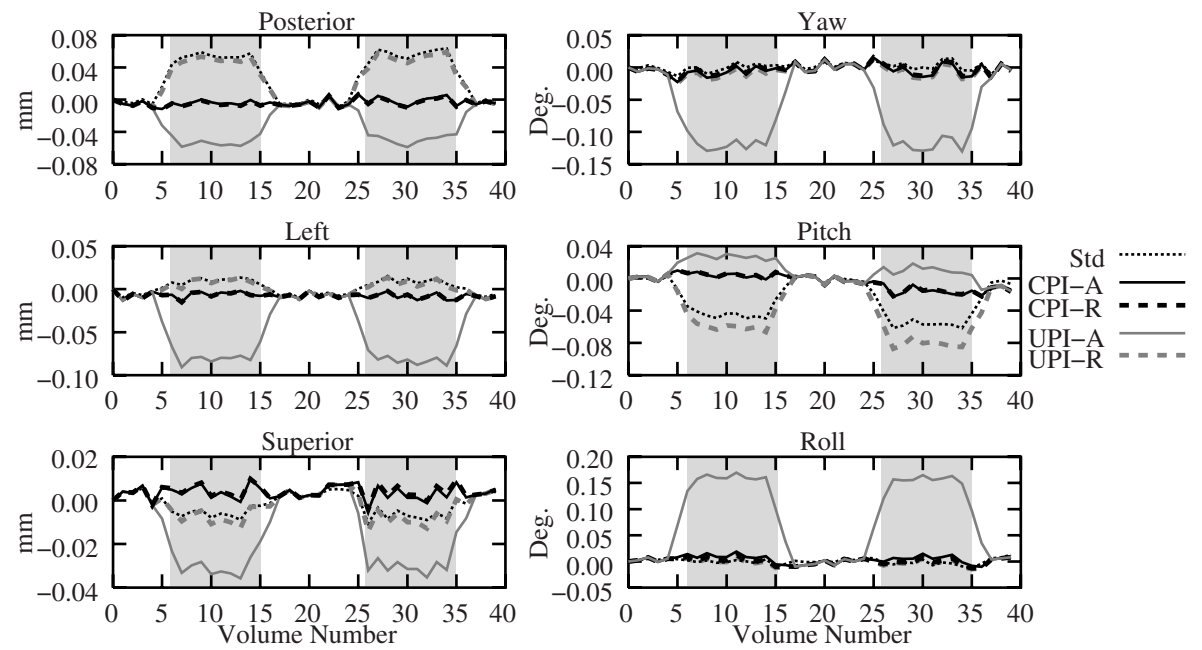

Fig. 2. Motion error plots for a typical dataset with activation and random motion. The shaded ranges show when the activation is present in the dataset.

The false-positive and false-negative counts for the six analysis methods are shown in tables 1 and 22, respectively. Notice that the QR decomposition and CPI methods consistently exhibit very similar counts, all resulting in approximately two-thirds fewer false-positive activations and one-third fewer false negative activations than the standard least-squares algorithm on the datasets with activation. 
Table 1. False-positive activation counts for the six algorithms, averaged over 10 trials for each dataset type.

\begin{tabular}{l|rrrrrr}
\hline Dataset Characteristics & Std & QR & CPI-A & CPI-R & UPI-A & UPI-R \\
\hline activation, random motion & 571.5 & 186.3 & 188.4 & 187.0 & $3,877.4$ & 747.5 \\
activation, stim-corr motion & 617.9 & 205.6 & 203.0 & 203.9 & $14,932.1$ & 796.9 \\
no activation, stim-corr motion & 42.7 & 38.4 & 36.9 & 36.5 & $15,732.8$ & 36.8 \\
activation, no motion & 586.0 & 187.8 & 190.1 & 188.5 & 190.2 & 744.7 \\
\hline
\end{tabular}

Table 2. False-negative counts for the six algorithms, averaged over 10 trials for each dataset type. Results for the datasets without activation are not shown because those datasets cannot, by definition, have any false negatives.

\begin{tabular}{l|cccccc}
\hline Dataset Characteristics & Std & QR & CPI-A & CPI-R & UPI-A UPI-R \\
\hline activation, random motion & 643.3 & 427.9 & 429.8 & 435.5 & 452.0 & 605.8 \\
activation, stim-corr motion & 642.3 & 429.0 & 427.0 & 425.3 & 379.9 & 603.6 \\
activation, no motion & 651.1 & 432.3 & 422.1 & 431.8 & 421.0 & 611.1 \\
\hline
\end{tabular}

The UPI-R method performed well on the datasets with no activation, while the UPI-A method performed well on motion-free datasets. This behaviour demonstrates that if the first process applied in the UPI method removes most of the time-series variation, then the method will work well. However, the UPI methods performed poorly on datasets that contained a mix of both motion and activation.

\section{Discussion and Conclusions}

Simply iterating the registration and activation detection processing steps yields a simultaneous solution to both problems. However, since the combined leastsquares problem does not have a unique solution, these unconstrained paired iteration methods usually converge to undesirable solutions.

In order to steer toward the desired solution, an additional constraint can be enforced during each iteration. The constrained paired iteration technique in which the activation detection is performed first (CPI-A) was shown to be mathematically equivalent to the QR decomposition method. In practice, while the motion estimates for the CPI-A method are very close to those of the $\mathrm{QR}$ decomposition method, they are not exactly the same because their implementations on a finite-precision computer can cause small numerical differences to arise.

Testing on simulated datasets showed that the two CPI methods perform as well as the QR decomposition method. On datasets containing activation, the CPI and QR decomposition methods all yield about two-thirds fewer false- 
positive activations and one-third fewer false-negative activations than the standard approach.

The paired iteration methods can be implemented efficiently using almost entirely off-the-shelf programs. Although we designed a $\mathrm{C}++$ program to enforce the additional constraint, it is also possible to carry out this procedure using a gradient descent type method with basic Perl programming and AFNI commandline programs.

The superior accuracy and ease of implementation of the constrained paired iteration methods offers a viable and robust solution for fMRI registration. Evaluation of the paired iteration methods on in vivo datasets is currently underway.

\section{References}

1. Ramsey, N.F., van den Brink, J.S., van Muiswindle, M.M.C., Folkers, P.J.M., Moonen, C.T.W.: Phase navigator correction in 3D fMRI improves detection of brain activation: Qualitative assessment with a graded motor activation procedure. NeuroImage 8 (1998) 240-248

2. Hajnal, J.V., Myers, R., Oatridge, A., Schwieso, J.E., Young, I.R., Bydder, G.M.: Artifacts due to stimulus correlated motion in functional imaging of the brain. Magn. Reson. Med. 31 (1994) 283-291

3. Mathiak, K., Posse, S.: Evaluation of motion and realignment for functional magnetic resonance imaging in real time. Magn. Reson. Med. 45 (2001) 167-171

4. Morgan, V.L., Pickens, D.R., Hartman, S.L., Price, R.R.: Comparison of functional MRI image realignment tools using a computer-generated phantom. Magn. Reson. Med. 46 (2001) 510-514

5. Freire, L., Mangin, J.F.: Motion correction algorithms of the brain mapping community create spurious functional activations. In: Proc. Info. Proc. Med. Imag., Davis (2001) 246-258

6. Freire, L., Mangin, J.F.: Two-stage alignment of fMRI time series using the experiment profile to discard activation-related bias. In Dohi, T., Kikinis, R., eds.: Medical Image Computing and Computer-Assisted Intervention (MICCAI'02). Volume 2489 of LNCS., Tokyo (2002) 663-670

7. Orchard, J., Greif, C., Golub, G., Bjornson, B., Atkins, M.S.: Simultaneous registration and activation detection for fMRI. IEEE Trans. Med. Imag. (2003) (in press).

8. Cox, R.W.: AFNI: Software for analysis and visualization of functional magnetic resonance neuroimages. Computers and Biomedical Research 29 (1996) 162-173

9. Nelder, J.A., Mead, R.: A simplex method for function minimization. Computer Journal 7 (1965) 308-313

10. Press, W.H., Teukolsky, S.A., Vetterling, W.T., Flannery, B.P.: Numerical Recipes in C: The Art of Scientific Computing. Second edn. Cambridge University Press (1988)

11. Eddy, W.F., Fitzgerald, M., Noll, D.C.: Improved image registration using fourier interpolation. Magn. Reson. Med. 36 (1996) 923-931

12. Cox, R.W., Jesmanowicz, A.: Real-time 3D image registration for functional MRI. Magn. Reson. Med. 42 (1999) 1014-1018 